\title{
Quantitative Studies of Sequential Peripheral Nerve Fiber Diameter Histograms and Biophysical Implications
}

\author{
Spencer I. BeMent and Walter H. Olson ${ }^{1}$ \\ Bioclectrical Scicnces Laboratory, Electrical and Computer Engincering Department \\ The Uniacrsity of Michigan, Ann Arbor, Michigan 48109, and \\ Department of Elcctrical Engineering, The University \\ of Illinois, Urbana, Illinois 61801
}

Recia'ed January 24, 1977; revision received July 7,1977

\begin{abstract}
Peripheral nerve cross sections were analyzed for the variation in peripheral nerve histograms obtained along a single short segment of cat saphenous nerve. Individual myelinated fibers in one fascicle were traced through nine sequential scctions. Two mean fiber diameter histograms were obtained from the sequential cross sections: a "mean fiber diameter histogram" constructed from the arithmetic average of the number of fibers in each bin of each section, and a "histogram of mean fiher diameters" constructed from the mean diameter of each fiber traced through multiple sections. Compared to the individual sectional histograms, the number of fibers at the two extremes of the dianeter range was generally less and the mean histograms were smoother. The sma11diameter peak of the "histogram of mean fiber diameters" was enhanced 22 to $65 \%$ over the peak of the individual sectional histograms and $40 \%$ over the "mean fiber diameter histogram." These findings indicate that any single-cross section fiber diameter histogram may be a relatively poor quantitative approximation of the histogram of mean fiber diameters; particularly at the extreme diameters and at the peaks and valleys of the distribution. A computer-based model was developed to adjust single-section fiber diameter histograms to compensate for sampling error associated with sing'e-section histograms. The adjustment procedure enhanced the narrow small diameter peak and broadened the valleys in accordance with the model theory and the
\end{abstract}

Abbreviations: MFDH-mean fiber diameter histogram; HMFD-histogram of mean fiber diameter; Pdf-probability density function.

1 Supported by U.S. Public Health Service Grants NS-08470 and GM-01289. The authors wish to thank Mr. Thomas G. Sandercock for his assistance in the model formulation and computer programming aspects of this study. The help of Mr. Robert Kushler and Dr. William Ericson of the Statistical Research I.aboratory at the University of Michigan is greatly appreciated. 
experimental results. The biophysical implications of the results were exanined in terms of (i) the relationship between fiber diameter and conduction velocity, and (ii) the relationship between fiber diameter histograms and the neural compound action potential.

\section{INTROIDLCTION}

The nerve filjer dianeter histugran has been used as the anatonical basis for reconstruction of compound action potentials $(3,+, 11,13,17$, $18,23)$, in relating conduction velocity to fiber dianneter $(5,13,15,21$, $29,33)$ and in degeneration and regeneration studies $(8,31)$. Such studies usually require measurements of fiber diameter oibtained from fresh, teased nerve fibers or from whole nerves that have been fixed, stained, sectioned, and mounted on slides. The dianeter is measured as the thickness along the length of the teased fiber preparations or from montages of enlarged photographs of transverse sections of nerve bundles. The "dlanneter" is obtained from the cross sections of single fibers by various methods including the best approximation to circles of various diameters (9); maximum, mininum (14), and mean (28) dianeter measurements; area $(11,27)$ and perimeter $(25)$ measurements ; as well as by the use of particle size counters $(2,7)$ and flying spot scanner methods (16). The diameter histograms obtained with many of these methods are compared in another paper (25).

Fiber diameter histograms based on the filer cliameters present in a single cross section of a whole nerve or fascicle represent only one sample of the fiber diameters in that nerve. If each nerve fiber maintained a constant diameter along its length and if artifacts were not introduced by the histological procedures, then the single-section fiber diametcr histogram would be a good representation of the dianeter distribution. However, nerve fiber dimensions do vary along the length of the nerve. For peripheral nyyelinated fibers, the diameter increases at the perinoxlal regions, decreases at the nodes, and waxes and wanes along the internodes (32) even for filers that do not taper or branch along the length of interest. McDonald and Ohlrich (20) report a maximum variation in diameter measurements within an internode of $24 \%$ for central nyelinated fibers in dorsal and lateral columns. This variation is less than that reported for peripheral nerve fibers by Sunderland and Roche (31).

The longitudinal variation in fiber cliameter is generally ignored in most culantitative applications of the clata provided by fiber diameter histogranns. In particular, all the studies cited above hased on the simulation of compound action potentials used single-section fiber cliameter histogians as a basic model parameter. We have shown that the most scnsitive parameter in those simulation models is the fiber diameter histogran (24). Therefore, the question "How well does a single-section fiber diameter histogram 
represent the mean fiber diameter histogram?" is of great inportance for any studies based on single-section histograms.

The results given here represent our initial effort to answer the above question. We report a quantitative analysis of variations in peripheral nerve fiber diameter over short lengths and an examination of the significance of the fiber diameter variation in terms of two biophysical developments based on diameter histograms. The quantitative results provide a basis for correcting or adjusting single-section fiber diameter histograms which incorporates the statistical variations in fiber diameter derived from the data.

\section{METHODS}

Saphenous nerves from adult cats were fixed, removed, embedded, and sectioned. The following specific histological procedures were used to prepare nerve trunks for quantitatitve analysis. Immediately after the recording session, the cat was perfused through the heart with $0.25 \%$ glutaraldehyde in Millonig's phosphate buffer, followed by $3 \%$ glutaraldehyde in the same buffer, both at $p \mathrm{H} 7.4$ (22). Sections of the nerve at the positions of the stimulating and recording electrodes were removed and placed $30 \mathrm{~min}$ in the fixative. The tissue was postfixed in $1 \%$ osmium tetroxide in Millonig's phosphate buffer $(2 \mathrm{~h})$, dehydrated by $10 \%$ steps of ethanol (20 min each), and finally embedded in Epon 812. Transverse sections $1 \mu \mathrm{m}$ thick were stained with toluidine blue. Several serial sections were mounted per slide.

Photographic montages were constructed for various fascicles. The individual fibcrs in onc small fascicle (165 to 168 fibers) in nine 1 - $\mu$ m-thick sections (separated by $100 \pm 10 \mu \mathrm{m}$ ) were labeled and their perimeters were digitized from photomicrographic montages. Transparent overlays of Calcomp drawings of the digitized fibers in the nine cross sections were used to trace individual myelinated fibers through the nine sections.

Two factors associated with the process of tracing and digitizing the fibers require some explanation. First, a small percentage of the fibers could not be traced with reasonable certainty in all nine sections. Second, a few fibers (two to five) in each section were not digitized originally so that they could not be included in some of the computed data. The result of these two factors is that nine diameter values per fiber are available for about $89 \%(148 / 167)$ of the fibers in the fascicle. There are eight diameter values for 11 of the fibers and less than eight values for the remaining fibers. No fiber diameter values were elininated because of supposed artifacts, unusual myelin forms, elliptical cross sections, or nodal or perinodal sections. This worst-case philosophy was applied wherever possible in an effort not to bias the statistical methods. 
The digitized primeter data were processed to yield several estimates of equivalent outside fiber diameter (25). The results presented here were based on a diameter estimate from the cross-sectional area of each fiber, $D=[4 \times \mathrm{area} / \pi]^{\frac{1}{1}}$. Thus $D$ was the diameter of a circle with area equal to the area bounded by the fiber perimeter.

Computer programs were developed to process the digitized data, to perform various statistical analyses (mean, standard deviation, regression), to plot some of the fiber diameter histograms and to adjust single-section histogram data based on the statistical analysis of the sequential-section data. The details of the last procedure are presented below.

There are four possible sources of bias and artifact in the study presented here. Estimates of error are given wherever possible.

i. Dimensional changes in the tissue preparation associated with the histological methods. The mitochondria and myelin were seen to be intact on examination under an electron microscope, however, slight shrinkage of the axoplasm in the large fibers was observed. The overall shrinkage for myelinated fibers that occurs during fixation and dehydration is estimated at 4 to $6 \%$ in the methods developed for electron microscopy $(6,22,29)$.

ii. Errors associated with the accuracy of the optical magnification determination. Four ocular reticule readings for each fascicular section were compared with measurements from the corresponding photomicrograph. The optical magnification ranged from about 0.5 to $1.1 \%$ on either side of the mean values for the nine sections. These mean values were used to compute the scale factors (micrometers/inch) used in conjunction with the digitized sectional data.

iii. Errors associated with the digitization and computational process. The same fascicle was digitized 8 months later to examine the repeatability of the digital perimeter measurements. The mean difference in diameter based on area computations was $0.07 \mu \mathrm{m}$ with a standard deviation of $0.12 \mu \mathrm{m}$ (see Fig. 1B).

iv. Errors associated with the filer identificalion process. Each of the nine sections could have been no more than 110 nor less than $90 \mu \mathrm{m}$ apart. However, the general shape of these sequential fascicular cross sections did change somewhat and fibers could change their relative positions between the sampled sections. Therefore, the process of identifying fibers from section to section becomes a matter of judgment based on pattern recognition aspects of fiber positions, sizes, and groupings. The tendency for large fibers to remain large and small fibers to remain small is an important factor in the decision process. A fiber is "lost" in the identification process when it cannot be identified with confidence, based on examination of the adjacent cross sections or when there is more than one candidate for a particular fiber in any given cross section. Occasionally the identification of a fiber was aided by microscopic examination of several adjacent $1-\mu \mathrm{m}$ thick sections. In general, fibers that had mean diameters with relatively large standard deviations (Fig. 4) either had one or two unusually large or small diameter values out of the nine, or stepped up or down in diameter somewhere along the nine cross sections. Waxing and waning of the diameter values was also 
apparent with some fibers whereas the values for other fibers seemed to vary little throughout the nine sections.

The fact that fiber size was one of the factors used in the process of tracing the fibers from section to section probably biases the result in favor of smaller standard deviations. On the other hand, any mistakes in the fiber tracing procedure would tend to increase the standard deviation. All fibers with "large" standard deviations were reexamined and in about one-half of these cases a new decision (identification) was made. These reevaluations altered one or more values for those fibers and for one or more fibers in the immediate vicinity. However, it should be pointed ont that such new decisions occurred for less than $10 \%$ of the fibers and that although such decisions increased the correlation coefficient slightly they did little to change the basic regression line used to describe the variation in fiber diameter as a function of fiber diameter. This regression line was already well defined by the large percentage of the fibers for which the initial decision was unaltered.

The uncertainty associated with the fiber identification process could be reduced by smaller sectional separation distances, e.g., 25 to $50 \mu 111$. Increasing the number of sections should increase the reliability of the diameter variance associated with each fiber.

\section{RESULTS}

A low-power photograph of a cross section of the saphenous nerve studied here is shown in Fig. 1. The small fascicle used in the sequential studies is indicated by the arrow. The Calconp plot for that fascicle is also shown. One hundred sixty-five to one hundred sixty-eight fiber cross sections were identified in the photomicrographs of the nine sequential sections.

Mean Fiber Diameter Histograms. The fiber diameter histograms for the nine sections of the fascicle used in this study are shown in Fig. 2. The largest diameter $(16 \mu \mathrm{m})$ appears in the seventh section. In general, the histograms have apparent peaks at $4.5,7.25$ to 8.75 , and 8.75 to 12.75 $\mu \mathrm{m}$. A relative minimum always appears somewhere between 5.75 and 7.75 $\mu \mathrm{m}$. The histogram that is the arithmetic mean of the nine histograms of Fig. 2 is given in Fig. 3A and is called the "mean fiber diameter histogram" (MFDH). An analysis of variance (anova, $\alpha=0.05$ ) indicates that the small-diameter peak is in the 4.25 to $4.75-\mu \mathrm{m}$ bin but that there is no statistical difference between mean values in the diameter range 5.75 to $7.25 \mu \mathrm{m}$ (histogram valley) or 7.75 to $11.75 \mu \mathrm{m}$ (histogram largefiber peak). As might be expected, the histogram in Fig. 3A is much smoother than any of the individual histograms in Fig. 2. Also, in general 


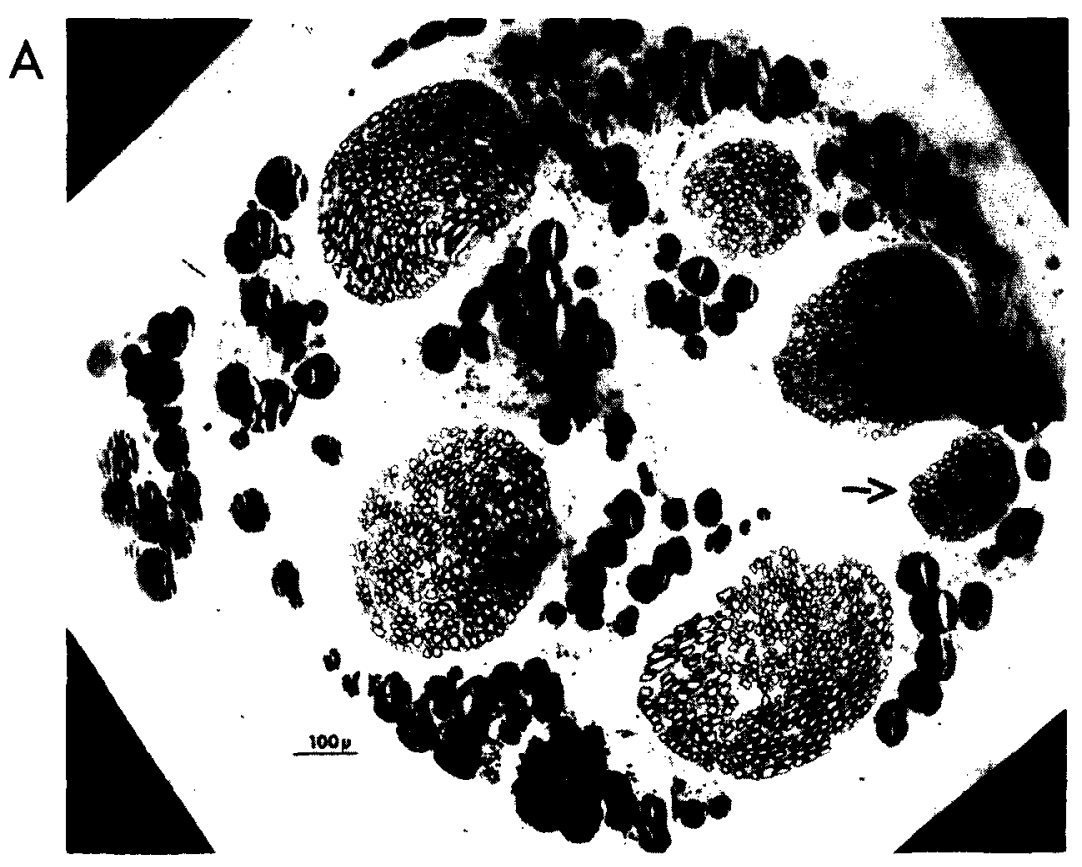

B

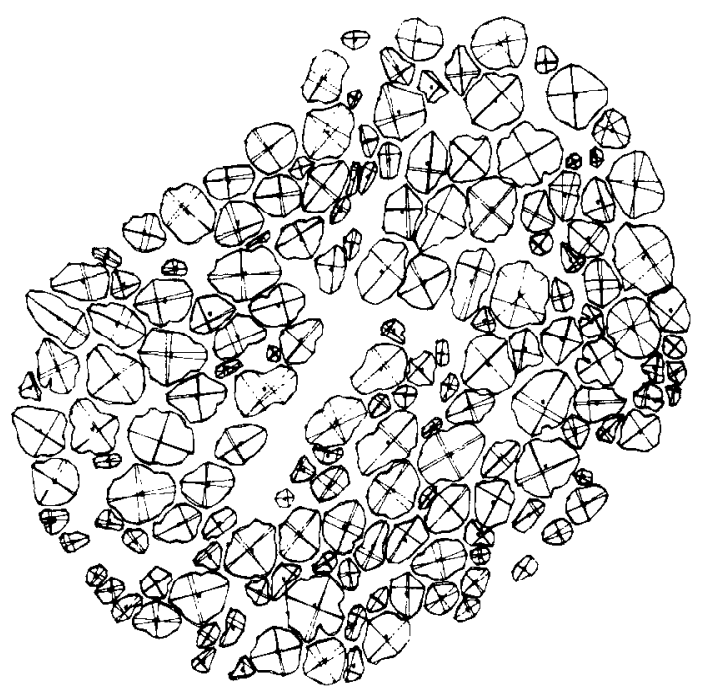

FIG. 1. A-Nerve and fascicular cross scctions, I.ow-power photomicrography of $1-\mu m-$ thick cross section of the cat saphenous nerve used in these studies. The arrow indicates the small fascicle analyzed in nine sequential sections. B-A Calcomp drawing of the fiber perimeters for the fascicle in $\mathrm{A}$. The lines across each fiber are the maxinum major and minor chords found by computer analysis of two independent series of perimeter coordinates. The actual plotted fascicle fits nicely on $8.5 \times 11$-in. $(21.6 \times$ 27.9-cm) paper. 

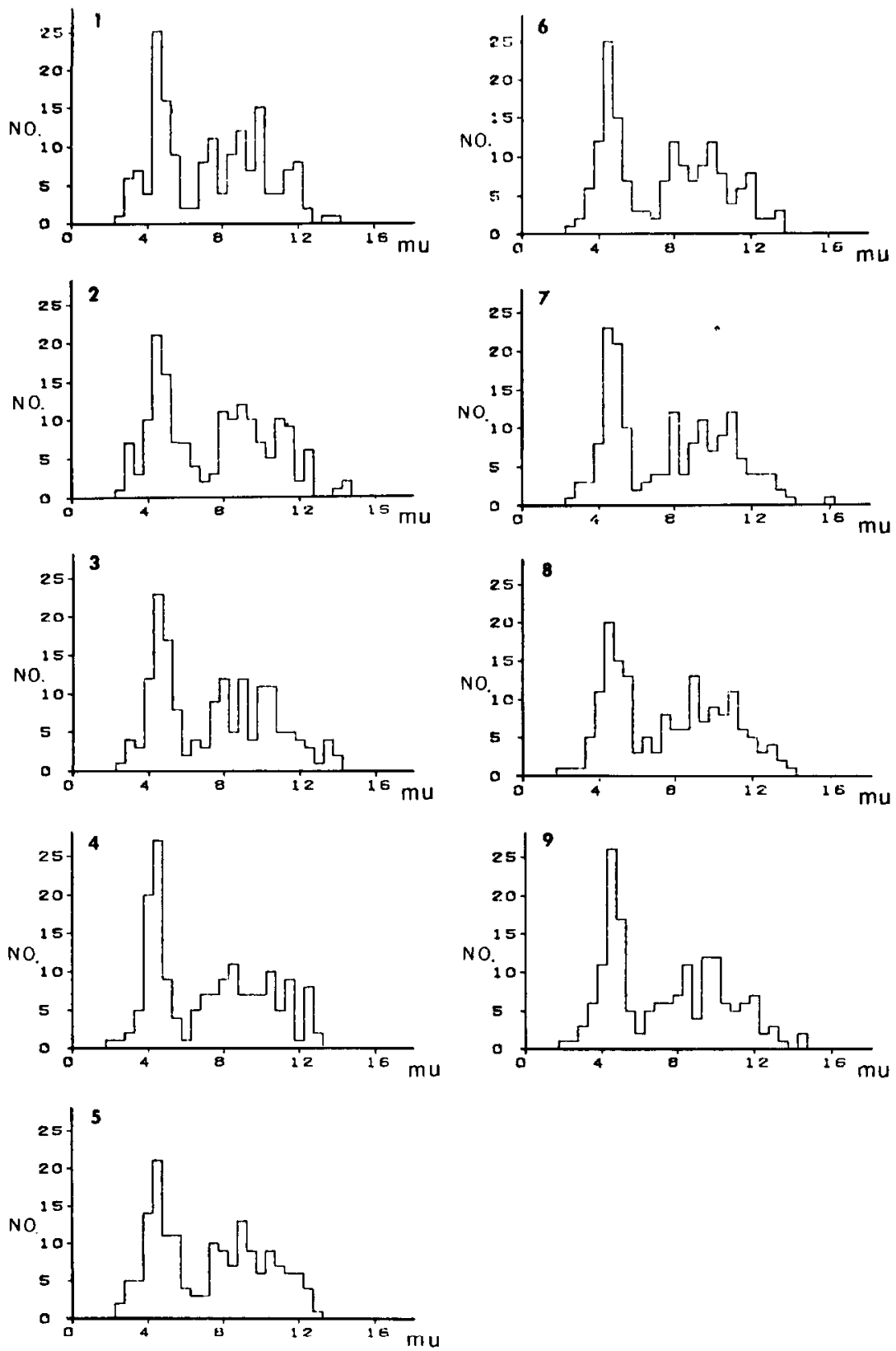

FIG. 2. Fiber diameter histograms for the nine 1- $\mu \mathrm{m}$ sections of the small fascicle of cat saphenous nerve used in this study. The sections were 90 to $110 \mu \mathrm{m}$ apart along the length of the nerve. 


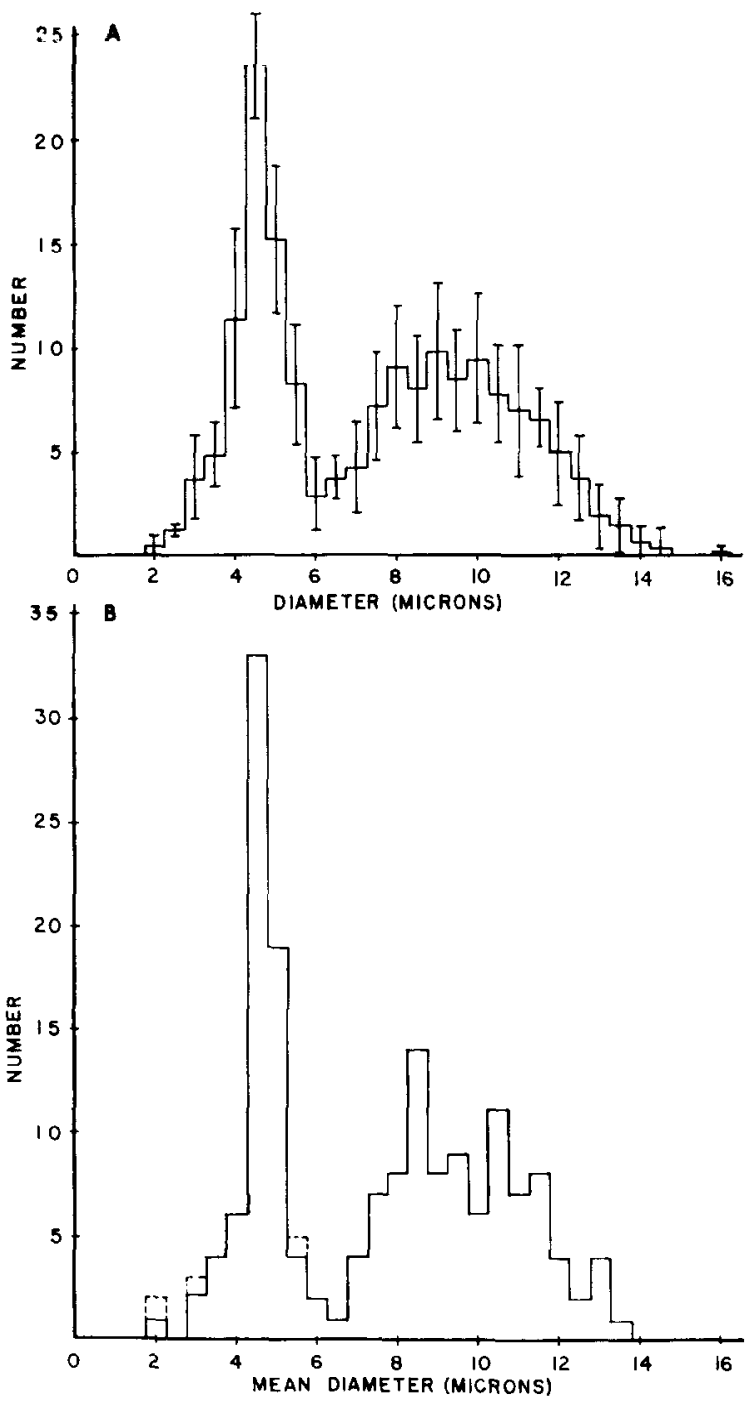

FIG. 3. Two different histograms based on sequential section data. A-Mean fiber diameter histogram (MFDH). This histogram is based on the mean of the number in each of the bins of the nine fascicular histograms shown in Fig. 2. The bars represent the standard deviation associated with the nine bin values. $\bar{N}=165.5$. B-Histogram of mean fiber diameters (HMFD). This histogram is based on the mean dianelers ior the single fiber traced through sequential cross sections. Of a total 168 fibers, 150 had values for all nine cross sections, 11 for eight cross sections, 2 for seven cross sections, 1 each for six, five, and four cross sections, and 2 for three cross sections. Refer to Methods for an explanation of the missing values. The dashed lines indicate diameters computed from less than five cross sections. 


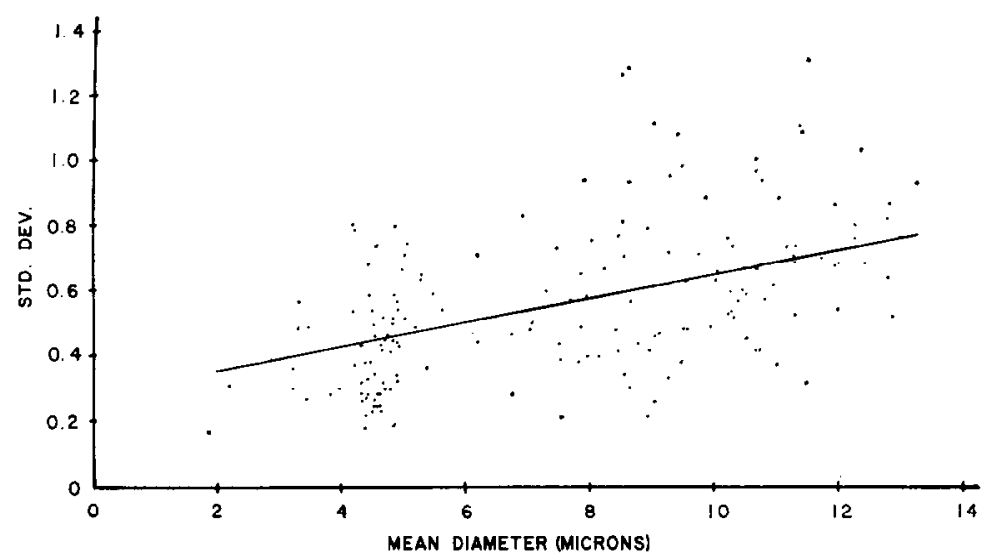

FIG. 4. Plot of standard deviation versus mean diameter. Each point is the mean and the standard deviation computed from nine single-section diameter determinations. One aberrant point $(9.24 \pm 2 \mu \mathrm{m})$ has been omitted on the plot and in the regression computations. The regression equation is $\mathrm{SD}=0.037 \times$ mean diameter +0.281 , with $r=$ 0.50 , based on 167 values.

the tails of the mean distribution are less pronounced than for the individual histograms. For example, the contribution of the single $16-\mu m$ fiber recorded in Fig. 2, section 7 , is relatively insignificant in the mean fiber diameter histogram.

Histogran of Mean Fiber Dianeters. The diameter values obtained for each fiber traced through as many as nine sections were used to compute a mean fiber diameter value for each of the 168 fibers. Chauvenet's criterion (35) was used to discard any diameter value from the nine-section sample whenever its deviation from the mean was so large that the probability of occurrence of such a deviation was less than $(2 N)^{-1}$. No more than one value was discarded per sample. The resultant niean values are represented by the "histogram of mean fiber diameters" (HMFD) shown in Fig. 3B. This histogram should be a better estimate of the "true" mean diameter histogram than that given in Fig. 3A because Fig. 3B is based on the statistical variation of the fiber diameters themselves rather than on the statistical variation of the single histograms represented by Fig. 3A.

Diancter Statistics for Traced Fibers. The standard deviation was computed from the diameter values recorded for each fiber traced through the multiple sections. Figure 4 is a plot of the standard deviation versus mean fiber diameter for those fibers. The regression line computed from these data is SD $=0.037 \times$ diameter +0.281 , with $r=0.50$ and $N=167$. There does not appear to be any merit in fitting these data with a higher-order equation. 
The data of Fig. 4 indicate a tendency for the standard deviation to increase with mean fiber diameter. This variation is consistent with the linited data of Matsumoto and Mori (19), who traced three cat superficial radial nerve fibers through 100 consecutive $1-\mu \mathrm{m}$-thick sections. Their mean diameter values of $2.3 \pm 0.17,7.5 \pm 0.4$, and $12.5 \pm 0.46 \mu \mathrm{m}$ fall below our regression line.

Single-Section Tersus Mean Fiber Diancter Histograns. How are single-cross section histograms related to the mean fiber histograms? The fact that the single-cross section listogranis vary from section to section is illustrated in Figs. 2, 3A. However, when the process of histogram construction from a single cross section is considered carefully, four important details become evident.

i. The filbers that contribute to a particular histogram bin are those fibers that happen to be in that particular dianeter range in that cross section. Therefore any measured diameter may actually be associated with a fiber whose moan diameter is smaller or larger than the measured diancter in that cross section.

ii. Unusually large single section fiber diameters are associated with fibers whose mean cliameter is actually smaller, and unusually small singlesection fiber cliameters are associaterl with fibers whose mean diameter is actually larger. In other words, in any single-section histogram the extreme diameter values represent contributions from the tails of the mean dianeter distributions for those fibers. Thus the number of filjers at the extrentes of the single-section disributions overrepresents the number of fibers that have these mean diameters. The largest fiber in the histogram of nean fiber diameters is $13.3 \mu \mathrm{m}$ whereas seven of the nine sectional histograms include diameters larger than $13.3 \mu \mathrm{m}$.

iii. Bins that contain low numbers of fibers at intermediate diameters also represent to some extent contributions from the tails of adjacent mean diameter distributions and thus overrepresent the number of fibers with mean diameters in those regions. The number of fibers in the $6-$ to 7 - $\mu \mathrm{m}$ valley of all sectional histograns is greater than the number of fibers in the same valley of the HMFD by 22 to $267 \%$.

iv. Because there is a fixed number of fibers in each cross section, the overrepresentation of fibers at the extremes and valleys implies that the number of fibers at the peaks and certain other dianeters is underrepresented. The small-diameter peak of the IIMFD is larger than the peak of all the single-section histograms by 22 to $65 \%$.

The important conclusion to be drawn from this analysis is that singlecross section fiber dianeter histograms may be a relatively poor quantitative approximation to the histogram of mean filer cliameters, particularly at the extreme diameters and at the peaks and valleys of the distribution. More- 


\section{TABLE 1}

Comparison of the Major Characteristics of the Mean Fiber Diameter Histogram (MFDH, Fig. 3A) and the Histogram of Mean Fiber Diameters (HMFD, Fig. 3B)

\begin{tabular}{lcc}
\hline \multicolumn{1}{c}{ Characteristic } & MFDH & \multicolumn{1}{c}{ HMFD } \\
\hline Diameter range $(\mu \mathrm{m})$ & $1.75-16.0$ & $1.8-13.3$ \\
Small-fiber peak $(\mu \mathrm{m})$ & $4.25-4.75$ & $4.25-4.75$ \\
Large-fiber peak $(\mu \mathrm{m})$ & $7.75-11.75$ & $8.25-8.75,10.25-10.75$ \\
Valley range $(\mu \mathrm{m})$ & $5.75-7.25$ & $5.75-6.75$ \\
Valley minimum $(\mu \mathrm{m})$ & $5.75-6.25$ & $6.25-6.75$ \\
No. of fibers in 4.5- $\mu \mathrm{m}$ bin & 23.44 & 33 \\
Total No. of fibers & 165.56 & 168 \\
Percentage less than $6.5 \mu \mathrm{m}$ & 42.9 & 44.0 \\
\hline
\end{tabular}

over, the histogram of mean fiber diameters is probably the actual histogram of interest in almost all quantitative anatomical, physiological, and biophysical applications of fiber diameter histograms.

Mean Fiber Diameter Histogram versus Histogran of Mean Fiber Diamcter. The two mean histograms have certain basic similarities. The small-

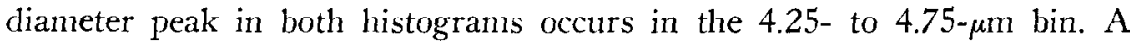
valley between the small-diameter and large-diameter groups exists between 5.75 and $6.75 \mu \mathrm{m}$. The principal differences between the two mean histograms are (i) the wider diameter range for the MFDH, (ii) the larger small-fiber diameter peak value of the HMFD ( $\sim 40 \%$ larger), and (iii) the two distinct large-diameter peaks in the HMFD compared to the broad peak $(7.75$ to $11.75 \mu \mathrm{m})$ in the MFDH. Table 1 is a comparative tabulation of the major characteristics of the two mean diameter histograms.

Mean Fiber Diameter Histograms from Single-Section Histogranis-A $M o d c l$. The sequential-section histograms and the two histograms of mean fiber diameter provide the basis for the development of a model to predict mean fiber diameter histograms from single-section histograms. The basic approach is that the number of fibers in any single-section histogram bin is the number of fibers whose mean diameter is actually within that diameter bin, plus a number of fibers of other mean diameters whose sampled diameter places them in that bin, ninus a number of fibers of that mean diameter whose sampled diameter places them in other bins.

The diameter of a fiber along its length, assuming no tapering or branching, can be approximated by some mathematical distribution. The data of Matsumoto and Mori (19, Fig. 2) indicate that this distribution may be approximated by a gaussian probability density function (Pdf),

$$
f(d)=e^{-(d-m) 2 / 2 \sigma^{2}} /(2 \pi \sigma)^{\vdots},
$$


where $d$ is a gaussian random variable with mean $m$ and variance $\sigma^{2}$. The chi-square values computed from their data was used to test the hypothesis that the population Pdf is gaussian. The gatussian hypothesis was not rejected at the $5 \%$ level of significance for the computed $\chi^{2}=6.23$, df $=14$. The chi-square value also indicates that there is a 95 to $97.5 \%$ probability that the differences between the Matsumoto and Mori distribution and a truly gaussian distribution are from random variation. Therefore, based on the serial-section data of Matsumota and Mori, we will assume that for any single-cross section histogram the diameter of each fiber is a gaussian random variable. We will also assume that the diameter of each fiber varies independently of the other filers.

As a first approximation, the area represented by each bin (number $x$ bin width) in a single-section histogram is the summation of the area contributed by fibers with mean diameters within that bin width and other areas contributed by fibers with mean cliameters outside that bin width, as indicated in Fig. 5. Therefore, if $y_{i}$ is the measured ordinate value for the $i$ th bin in an $n$-bin cross-sectional histogram, then $y_{i}=a_{i 1} y_{1}^{\prime}+\cdots+$ $a_{i j} y_{j}^{\prime}+a_{i n} y_{n}^{\prime}$, where $y_{j}^{\prime}$ is the true number of fibers in the $j$ th bin and $a_{i j}$ is the weighting function that represents the probability that fibers with mean values in the $j$ th bin contribute to the number of fibers in the $i$ th bin. Because there are $n$ of these equations, one for each of the $n$ bins in the cross-sectional histogram, this system of $n$ equations in $n$ unknowns can be solved when the weighting functions are known. The weighting functions can be approximated by the percentage of area under a gaussian Pdf that

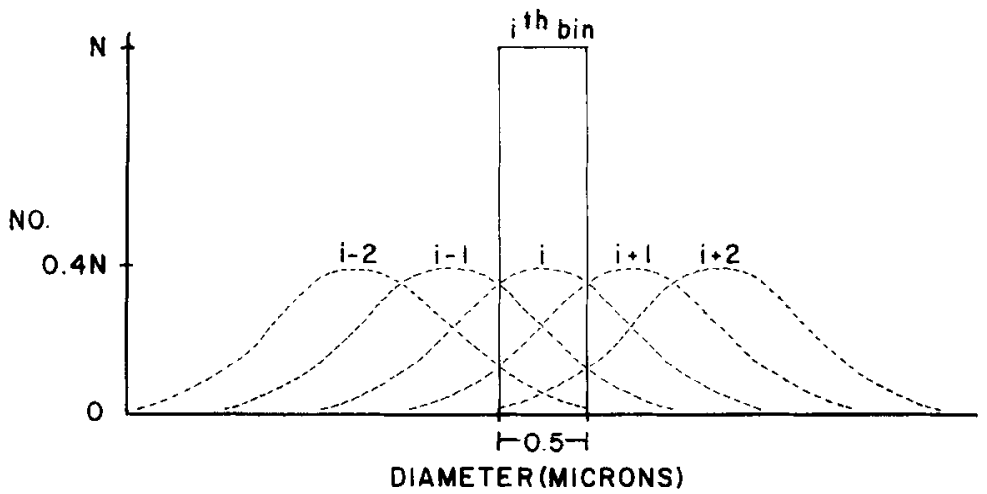

FIG. 5. Stylized example of summation of gaussian distributions. The gaussian bin fiber diameter distributions are added to result in a value $N$ for any particular discrete ith fiber diameter bin. The standard deviation for the spread of the "true" nean fiber diancter into other bins is fixed at $0.5 \mu \mathrm{m}$ in this artificial example. The area under ath gaussian distribution is proportional to $Y^{*}{ }_{1}$, the number of fibers actually in the th bin of the true mean histogram. $Y^{\prime}{ }_{i}$ is assumed constant in this example. 
represents the spread of the true mean fiber diameter into other bins. For example, we will assume that the variation in fiber diameter for fibers with a mean diameter of $y_{i} \mu \mathrm{m}$ is represented by a gaussian Pdf with standard deviation $0.5 \mu \mathrm{m}$. We will also assume for simplicity that the histogram bin width is equal to the standard deviation. Then $38.30 \%$ of the fibers with a true mean diameter of $y_{i} \mu \mathrm{m}$ will fall in the range $y_{i} \pm 0.25 \mu \mathrm{ml}( \pm 0.5 \sigma)$, $24.17 \%$ from $y_{i}+0.25$ to $y_{i}+0.75$ and from $y_{i}-0.75$ to $y_{i}-0.25 \mu \mathrm{m}$ $(0.5$ to $1.5 \sigma), 6.06 \%$ from $y_{i}+0.75$ to $y_{i}+1.25$ and from $y_{i}-1.25$ to $y_{i}-$ $0.75 \mu \mathrm{m}$ ( 1.5 to $2.5 \sigma)$. The small remaining percentage $(1.24 \%)$ of fibers falls in the other bins greater than 2.5o. This example is shown in Fig. 5.

A computer program was developed to adjust single-cross section histograms which takes into account variations in fiber diameter with length as described by a gaussian Pdf. By definition, the single-cross section histogram is referred to as the measured fiber histogram and the correcter histogram as the adjusted fiber histogram. The system of simultaneous equations is shown below (one equation for each histogram bin):

$$
\begin{aligned}
& y_{1}=a_{11} y^{\prime}{ }_{1}+a_{12} y^{\prime}{ }_{2}+\cdots+a_{1 n} y^{\prime}{ }_{n}, \\
& y_{2}=a_{21} y_{1}+a_{22} y^{\prime}+\cdots+a_{2 n} y_{n}, \\
& \vdots \\
& y_{n}=a_{n 1} y^{\prime}{ }_{1}+a_{n 2} y^{\prime}{ }_{2}+\cdots+a_{n} y^{\prime}{ }^{\prime},
\end{aligned}
$$

where $y_{i}=$ ordinate value of bin $i$ in the measured fiber histogram, $y_{i}^{\prime}=$ ordinate value of bin $i$ in the adjusted fiber histogram (unknown value), and $a_{i j}=$ probability that fibers with mean diameter within bin $j$ will be measured with diameter of bin $i$. The $a$ 's are calculated as follows:

$$
a_{i j}=\int_{D_{j-\alpha}}^{n_{j+\alpha}+\alpha} f_{j}(x) d x \quad(1<j<n),
$$

with $\sum_{i=1}^{n} a_{i j}=1$. The bin width is $2 \alpha, D_{j}$ is the diameter represented lry bin $j$ (bin $j$ includes any fiber with diameter from $D_{j}-\alpha$ to $D_{j}+\alpha$ ), and $f_{j}(x)$ is a gaussian Pdf with mean value at the center of bin $j$ and standard deviation $\sigma_{j}$ for that value.

As previously mentioned, the Pdf for the diameter of a single fiber sampled at random points along the nerve can be approximated by a gaussian distribution. In the computer solution, the standard deviations of the gaussian Pdf for the diameters of the smallest and largest bins are specified and the standard deviations for the intermediate bins are obtained by linear interpolation.

The system of equations can be represented by the matrix equation $\bar{y}=\bar{A} \cdot \bar{y}^{\prime}$, where $\bar{A}$ is an $n \times n$ matrix of the weighting functions $a_{i j}, \bar{y}^{\prime}$ is a column matrix of the bin values for the adjusted fiber histogram, and $\bar{y}$ is a column matrix of the known bin values for the measured histograms. 
The matrix solution of these equations yields the predicted values for the mean fiber histogram.

The program was checked by generating a gaussian "measured" histogram ( $\bar{y}$ values) and solving for the "adjusted" histogram ( $\bar{y}^{\prime}$ values) using the mean and standard deviation of the measured histogram. The adjusted histogram matched the measured histogram within the roundoff error.

The experimental clata can be used to illustrate this histogram adjusting method. The single fascicle histograms of Fig. 2 represent a small sample of nerve fibers in saphenous nerve. Because of the small sample size, the number of fibers in any bin of these histograms is subject to a larger sampling error than if the sample size were all the filers in that nerve,
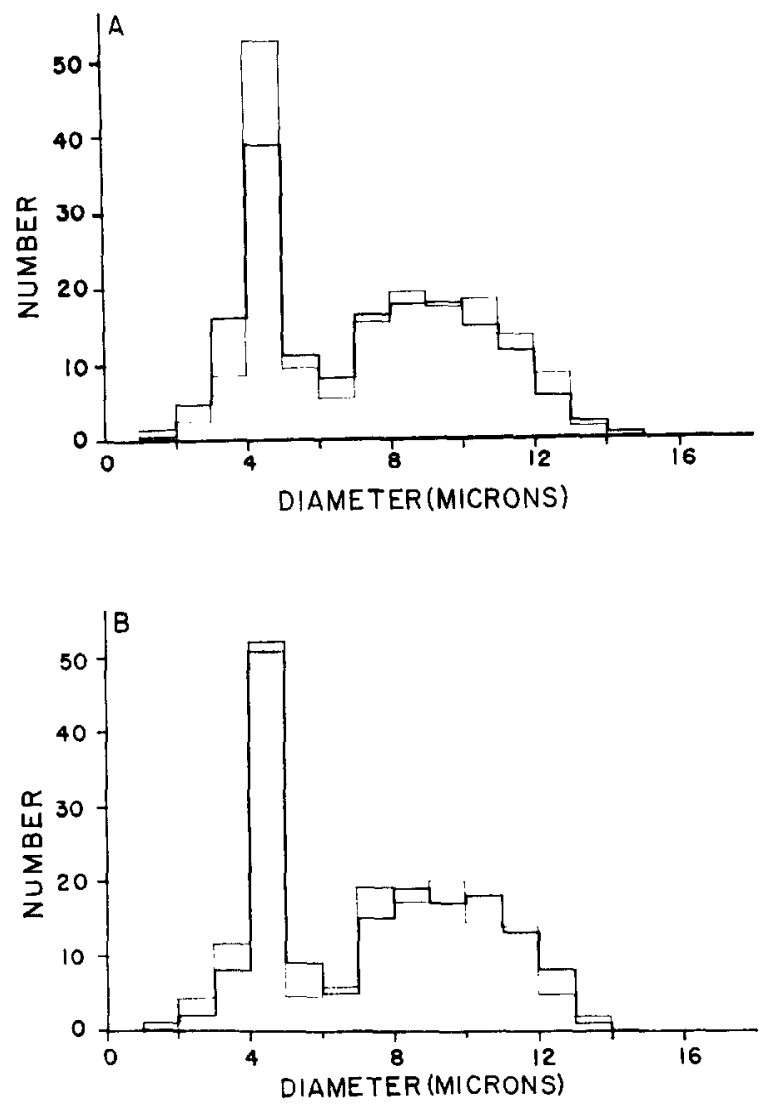

Fig. 6. Adjusted mean fiber diameter histogram (MFDH). A-The result (fine line) of adjusting the MFDH (heavy line). The regression equation from Fig. 4 was used to represent the variation in fiber diameter as a function of fiber diameter, i.c., the standard deviation of the gaussian distribution of Fig. $5 . \mathrm{B}-\mathrm{A}$ comparison of the adjuster MFDH (fine line in $A$ ) with the histogran of mean fiber diameter (HMFD). 
approximately 2500 fibers. However, the mean fiber diameter histogram of Fig. 3A represents much less sampling error than does any single sequential histogram. Therefore, we will assume that the mean histogram (MFDH) of Fig. 3A is a reasonable approximation to the single-section histogram we would get if the number of fibers in any of our single-section samples was larger by about an order of magnitude.

The histogram adjustment procedure was applied to the MFDH of Fig. 3A. The regression line fitted to the data of Fig. 4 was used to describe the standard deviation of the mean fiber diameter as a function of fiber diameter. The result of adjusting the $\mathrm{MFDH}$ is compared with the unadjusted histogram in Fig. 6A. The adjustment procedure enhanced the small-fiber diameter peak by $37 \%$, depressed the valley region from 5 to $7 \mu \mathrm{m}$, and eliminated fibers with cliameters greater than $14 \mu \mathrm{m}$.

The adjusted MFDH is compared with the HMFD in Fig. 6B. The "match" is quitc good, with perhaps the major difference being that the number of fibers in the 5- to $6-\mu \mathrm{m}$ bin is not as small as in the HMFD.

The comparison shown in Fig. 6B seems to indicate that the model developed to account for the differences between single-section histograms and the more highly desirable HMFD is reasonable. The model does predict the enhancement of strong narrow peaks, the depression of valleys, and the narrowing of the range of fiber diameters seen in the $\mathrm{HMFD}$ with respect to the MFDH. The same results should be apparent when the model is used to adjust single-section histograms based on large numbers of fibers such that the sampling error associated with any bin is relatively small.

\section{DISCUSSION}

\section{Sequential-Section Histograms}

The results presented here appear to be the first comparisons of fiber diameter histograms from sequential sections of the same nerve. The histograns in Fig. 3 represent alternative methods for extracting a "mean" fiber diameter histogram from such multiple sections.

It is apparent from the sectional histograms of Fig. 2 that certain basic features such as the small-fiber diameter peak are preserved in all the histograms. However, the range of fiber diameters, the location of the other peaks, and the diameter range of the valley vary noticeably. Surprisingly, none of the sectional histograns is statistically different from any other of the sectional histograms, according to the Kolmogorov-Smirnov two-sample test (30) with $\alpha=0.05$. This test is a test of whether or not two independent samples have been drawn fron populations with the same distribution. The two-tailed test is sensitive to any kind of differences in the distributions 
from which the two samples were drawn-differences in location, in skewness, in dispersion, etc.

\section{Fiber Dianneter Variation with Length}

The standard deviations presented in Fig. 4 are larger than those found in three cat superficial radial fibers analyzed by Matsumoto and Mori (19). Although others have noted variations in fiber diameter with length they rarely report the mean and standard deviation values. Hursh (15) reported $6.5 \pm 0.47 \mu \mathrm{ml}$ for a single fiber traced through 100 consecutive $6-\mu \mathrm{m}$ paraffin sections of cat saphenous nerve. His value lies slightly below the regression line of Fig. 4. When the data reported by Sunderland and Roche (31) for 40 consecutive 10- $\mu \mathrm{m}$ sections of opossum median nerve fiber (their Table IV) are analyzed on the basis of outside diameter, the result is a mean diameter of $12.5 \pm 1.6 \mu \mathrm{m}$. Their standard deviation is more than double that predicted by the regression line of Fig. 4 .

The scatter of the data points in Fig. 4 reflects several known sources of experimental error in addition to the actual diameter variation from the nonuniformity of the fibers. The error contributed by shrinkage probably is not an important factor because the evidence available indlicates that shrinkage is relatively uniform for the structures we are measuring. The experimental error present in the magnification factor and the digitization process should make only minor contributions to the variance of Fig. 4.

The variance introduced by computing diameter from perimeter data may be appreciable but it cannot be estimated from the limited number of fiber cross sections available. If more cross sections were available per fiber the data could be examined for the cffect of diameters computed from noncircular cross sections on the resultant mean diameter values.

The plane of each cross section is such that some fibers are always sectioned obliquely. Therefore, the perimeter and thus the computed area and fiber diameter for obliquely sectioned fibers are larger than would be the case for transversely sectioned fibers. However, as noted by Fraher (10), if the plane of section of a cylindrical fiber is angled away by $25^{\circ}$ or less on either side of transverse, the observed circumference value exceeds the transverse value by $5.2 \%$ or less. A $5 \%$ increase in circunference of both a circle and an ellipse with major axis twice the minor axis results in about a $5 \%$ increase in diameter. Therefore, fibers sectioned at oblique angles less than $25^{\circ}$ probably have increased diameter values of $5 \%$ or less.

The possible effects of oblique sections were simulated as follows. An artificial data set of nine values from 3.9 to $5.5 \mu \mathrm{m}$ in $0.2-\mu \mathrm{m}$ increments save a mean of 4.7 with $\sigma= \pm 0.5+8$. When five of the diameter values including the extreme values were increased by $5 \%$ to simulate a severe (obliçueness conrlition, the mean was t.s with $\sigma= \pm 0.575$. This rather 
severe obliqueness test increased $\sigma$ by about $4 \%$. When the same procedure was used on a data set from 9.7 to 12.1 in $0.3-\mu \mathrm{m}$ increments, $\sigma$ increased by $9 \%$. Therefore, it seems reasonable to assume that the presence of fiber obliqueness could contribute less than $10 \%$ of the variance present in the values of Fig. 4.

The location of the sections along a given fiber is important in our small sample because the nine values are not taken at the same relative points along each fiber with respect to the nodes, internodes, and perinodal regions. This type of variation is obvious when one examines the raw data because within the nine values there may be one or two "large" or "small" values that contribute greatly to the standard deviation and the mean diameter values. Chauvenet's criterion was used to reduce this effect.

There is variation introduced through the uncertainty associated with the identification of each fiber in tracing the "same" fiber through the nine sections where each section is 90 to $110 \mu \mathrm{m}$ from the preceding section. This is perhaps the single greatest source of experimental error that contributes to the wide range of standard deviations shown in Fig. 4. We know of no method to estimate how much of the data variance is contributed by such errors.

The scatter of the data in Fig. 4 could be reduced by analyzing more cross sections and by reducing the distance between cross sections. The results should be a reduction in the standard deviation for some fibers such that the data points in Fig. 4 would be lower and the regression line would have a lower value for the $y$ intercept. It is not possible to predict changes in the slope of the regression line.

\section{The Histogram Adjustment Approach}

The procedure for "adjusting" single-section histograms to reflect the statistical variation in filser cliameter appears to be a valid method to gain a better approximation of the true mean fiber diameter histogram when only one cross section is available. When the MFDH was adjusted with the statistical parameters derived from Fig. 4 the result was quite similar to the HMFD which is our best estimate of the true fiber diameter histogram. It should be pointcd out that the model developed to describe singlesection histograms predicts the major differences observed between the two mean histograms. The conceptual basis for the model appears sound and the results are consistent with the experinental clata. The actual values of standard deviations for the gaussian distributions associated with the mean fiber diameters are still in question. Futture studies based on increased numbers of more closely spacerl sequential sections should clecrease the scatter of the data of Fig. 4 and increase the accuracy of the values used for the standard deviations in the histogram adjustment procedure. 


\section{Biophysical Implications of the Results}

The results reported here have direct bearing on biophysical constants and equations that relate the anatomy of nerve trunks as reflected by the fiher dianeter histograns to the physiology of nerve trunks as reffected by nerve compond action potentials. Two bioflysical relations are discussed here.

Condution lolocity atersts liber liander. The most commonly accepted relation between conduction volocity and filser diancter is based on the work of Hursh (15). He found a slope of about 6 for the regression line fitting the scatter diagram of conduction velocity (meters per second) versus fiber diameter (micrometers) in cat peripheral nerves. His procedure of relating the largest fiber in a nerve bundle to the maximum conduction velocity (as determined by the shortest latency to the beginning of the (implied or otherwise) that the largest dianeter found in each cross section was a good representation of the diameter of that fiber between the stimulating and the recording site. However, as our clata indicate, the largest-dianeter fiber in any cruss section is generally larger than the mean diameter of any fiber thronghout the concluction distance. Therefore, the mean diameter value that should be related to any particular conduction velocity is probably sualler than that determined by Hursh.

Another problem arises in the literature because the data of Hursh have been misinterpreted. Textlooks $[(26)$, p. 9; (34), p. 59] and journal articles, e.g.. Ref. (1), use the slope of Hursh's data to assert in one form or another that the conduction velocity (meters per second) is equal to the fiber diameter (micrometers) times a conduction velocity constant, usually a constant value of about 6 . However, the regression line for Hursh's data does not go through the origin so that the "constant" from his data is actually different for various fiber diameters $[5.8(20 \mu \mathrm{m})$, $5 .+(8 \mu \mathrm{m})$, $4.7(4 \mu m))$ ]. Others have developed or used different constants for different ranges of fiber diameter without reference to this fact $(21,33)$.

The two points raised above provicle support for concluding that the relation between conduction velocity and mean fiber diameter is nonlinear and that the fiber diameter associated with any particular conduction velocity is smaller than would be predicted by Hursh's data. The result would be larger values for the conduction velocity constant than have been generally reported in the past.

The new data of Clark and Burgess (5) for cat medial and posterior articular nerves are the most extensive data on conduction velocity and fiber dianeter available. Their conduction velocity constant of 7.8 for the fastest conducting fibers in the posterior articular nerve is significantly larger than that predicted from Hursh's data (about 5.7 at $16 \mu \mathrm{m}$ ), and tends to support our prediction of larger conduction velocity constant values. 
Compound Action Potential a'ersus Fiber llameler. Monnhusic and multiphasic compound action potentials have heen simulated based on single-cross section fiber diameter listograms for peripheral nerves $(3,4$, $13,17,24,25)$. In all these simulations the histogram is mapped to the simulated action potential through some analytical expression that relates fiber diameter to conduction velocity. The results of our study are important on two counts:

i. The starting point (time) for the simulated compound action potentials is very dependent on the relation assumed between the maximum conduction velocity and the largest fiber diameter in the histogram. We have provided evidence in this study that the largest-diameter fiber in any single histogram is not a good estimate of the largest-diameter fiber in the nerve trunk. The mean diameter for the largest fiber in the fascicles analyzed is $13.3 \mu \mathrm{m}$ whereas the largest diameter in the nine sections varied between 13.1 and $15.9 \mu \mathrm{m}$. Based on our data, the large-fiber conduction velocity constant could be as much as $20 \%$ more than that predicted from the individual histograms. It now seem reasonable to assume that the largest 0.5 to $1.0 \%$ of the fiber diameters in any individual histogram actually represents cross sections of fibers of lesser mean diameter. Therefore, in the stimulation of compound action potentials we suggest that these fibers be redistributed into bins of slightly smaller diameter fibers. The actual redistribution procedure would depend on the character of a particular distribution at the larger-diameter end. For example, the results shown in Fig. 3B indicate that all the fibers with diameters greater than $13 \mu \mathrm{m}$ in the histograms of Fig. 2 should be lumped into bins less than or equal to that dianeter. The result would be a decreased latency for the compound action potential with little or no effect on the amplitude of the initial peak of the potential for conduction distances less than about $100 \mathrm{~mm}$. Likewise, the smallest $1 \%$ of the fiber diameters is probably overrepresented. However, the effect of errors at the smalldiameter end of the histogram would have minimal influence on the usual simulated compound action potential.

ii. 'The form of the single-cross section histogram is mapped in an inverse and nonlinear fashion into the form of the simulated action potential. However, the dependence of the standard deviation of the mean diameter on the mean diameter (Fig. 4) in conjunction with the fact that the fiber diameters are not uniformly distributed indicate that a single-cross section diameter histogram may be a relatively poor quantitative estimate of the histogram of mean fiber diameters. This conclusion is based on visual comparisons of each of the nine histograms of Fig. 2 with either of the mean histograms of Fig. 3A or B. The apparent "best" estimate for the mean fiber diameter histogram (Fig. 3B) differs appreciably from each of the diameter histogranns of Fig. 2. 


\section{RFFERENCIS}

1. BrMent, S. L., AND J. B. RANCK. 1969. A quantitative study of electrical stimulation of central myelinated fibers. Exp. Niurol. $24: 147-170$.

2. Berthold, C. H., and T. Carlstedt. 1973. Fixation and numerical estimation of myelinated nerve fibers during initial myelination in the cat. Neurobiology 3: $1-18$.

3. Buchthal, F., and A. Rosenfalck. 1966. Evoked action potentials and conduction velocity in human sensory nerves. Brain Res. $3: 1-122$.

4. CASEy, K. L., AND M. Blick. 1969. Observations on anodal polarization of cutaneous nerve. Brain Res. 13 : 155-167.

5. Cl.Ark, F. J., ANd P. R. Burgess. 1975. Slowly adapting receptors in cat knee joint: Can they signal angle? J. Nenrophysiol. 38: 1448-1463.

6. Dyck, P. J. 1966. Histological measurements and fine structures of biopsied sural nerve: Normal, and in peroneal muscular atrophy, hypertrophic neuropathy and congenital sensory neuropathy. Mayo Clin. Proc. 41: 742-774.

7. Dyck, P. J, AND F. H. LAmbekt. 1966. Numbers and diameter of nerve fibers and compound action potentials of sural nerve: Controls and hereditary neuromuscular disorders. Trans. Am. Nourol. Assoc. $91: 214-217$.

8. Dyck, P. J., J. A. Gutrecht, J. A. Bastron, W. E. Karnes, and A. V. D. Dalle. 1968. Histologic and teased fiber mcasurements of sural nerve in disorders of lower motor and primary sensory neurons. Proc. Mayo Clin. 43 : 81-123.

9. Espir, M. I. E., ANd D. T. C. Harding. 1961. Apparatus for measuring and counting myelinated nerve fibers. J. Ncurol. Nourosurg. Psychiatr. $24: 287-290$.

10. Fraher, J. P. 1972. A quantitative study of anterior root fibers during early myelination. J. Anat. $112: 99-124$.

11. Freide, R. L., AND T. Samorajski. 1967. Relation between the number of myelin lamellae and axon circumference in fibers of vagus and sciatic nerves of mice. J. Comp. Nourol. $130: 223-232$.

12. Glasser, H. S. 1955. Properties of dorsal root unmedullated fibers on the two sides of the ganglion. J. Gen. Physiol. $38:$ 709-729.

13. Gasser, H. S., And H. Grundfest. 1939. Axon diameters in relation to the spike dimensions and the conduction velocity in mammalian A fibers. Am. J. Physiol. $127: 393-414$.

14. Gutrecht, J. A., And P. J. Dyck. 1970. Quantitative teased-fiber and histological studies of human sural nerve during post-natal development. J. Comp. Ncurol. 138 : $117-129$.

15. Hersir, J. B. 1939. Conduction velocity and diameter of nerve fibcrs. Aner. $J$. Physiol. 127 : 131-139.

16. Kasvand, T., D. Isnor, ANd C. Romero-Sierra. 1970. Man atersus Compuicr in the Mcasurcment of Sizc Distribution of Nore Fibcrs, Mechanical Engineering Report ML-5, NRC No. 1, pp. 1-53. National Research Council of Canada.

17. Jandat, W. M., M. H. Clare, aNd G. H. Bishop. 1968. Reconstruction of myelinated nerve tract action potentials: An arithmetic method. Exp. Neurol. $22: 480-490$.

18. Lee, R. G., P. Ashby, D. G. White, and A. J. Aglayo. 1975. Analysis of motor conduction velocity in the human medial nerve by computer simulation of compound muscle action potentials. Electrocnccph. Clin. Ncurophysiol. 39: 225-237.

19. Matsumoto, A., and S. Mori. 1975. Number and diameter distribution of myelinated afferent fibers innervating the paws of the cat and monkey. Exp. Neurol. 48: 261-274. 
20. McDonaly, W. I., And G. D. Ohurich. 1971. Quantitative anatomical measurements on single isolated fibers from cat spinal cord. J. Anat. 110: 191-202.

21. McLeod, J. G., ANd S. H. Wray. 1967. Conduction velocity and fiber diameter of the median and ulnar nerves of the baboon. J. Nourol. Ncurosurg. Psychiatry $30: 240-247$.

22. Millonig, G. 1962. Further observation on a phosphate buffer from osmium solutions in fixation. Proc. 5th Int. Congr. Electron Microsc. 2 : P-8.

23. Olson, W. H. 1973. Peripheral Nere Compound Action Potcutials and Fiber Diameter Histograms. Ph.D. Dissertation. University of Michigan, Ann Arbor.

24. Olson, W. H., and S. L. BeMent. 1970. Compound action potentials : computer analysis of parameters that determine the waveform. $23 r d$ Ann. Conf. Eng. Mcd. Biol. $12: 46$.

25. Olson, W. H., S. L. BeMent, and M. D. Ross. Comparative estimates of fiber diameter for peripheral nerve histograms. J. Microsc. (submitted).

26. Plonsey, R. 1969. Bioelectric Phenomona. McGraw-Hill, New York.

27. Potts, A. M., D. Hodges, C. B. Shelman, K. J. Fritz, N. S. Levy, and Y. ManGALL. 1972. Morphology of the primate optic nerve. 2. Total fiber size distribution and fiber density distributions. Invest. Ophthalmol. 11: 980-1016.

28. Schlaepfer, W. W., and F. K. Myers. 1973. Relationship of myelin internode elongation and growth in the rat sural nerve. J. Comp. Neurol. $147: 255-266$.

29. Schnepp, G., P. Schnepp, and G. Spann. 1971. Analysis of peripheral nerve fibers in animals of different body size: 1 . Total fiber count, fiber size and nerve conduction velocity. Z. Zellforsch. $119:$ 77-98.

30. Sieger, S. 1956. Nonparanctric Statistics. McGraw-Hill, New York.

31. Sunderland, S., And A. Roche. 1958. Axon myelin relationships in peripheral nerve fibers. Acta Anat. $33: 1-37$.

32. Sunderiand, S. 1968. Nores and Ncroc Injurics. Livingstone, Edinburgh.

33. Whitehorn, D., J. F. Howe, M. J. Lessler, And P. R. Burgess. 1974. Cutaneous receptors supplied by myelinated fibers in the cat. I. Number of receptors innervated by a single nerve. $J$. Neurophysiol. 37 : 1361-1372.

34. Willis, W. D., and F. Grossman. 1973. Medical Neurobiology. C. B. Mosby, St. Louis.

35. Young, H. D. 1962. Statistical Treatment of Experimental Data. McGraw-Hill, New York. 